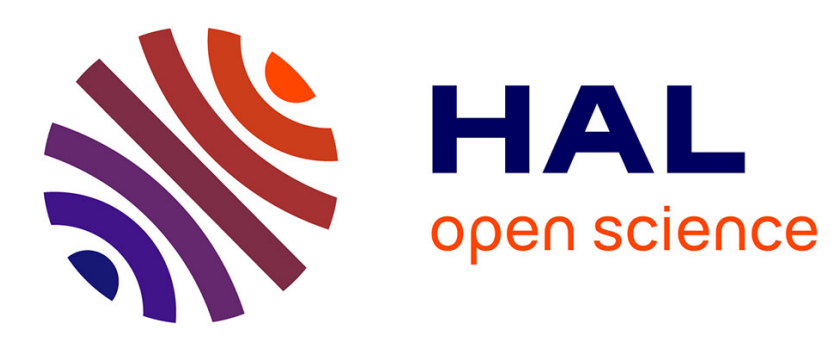

\title{
From Structurally Independent Local LTI Models to LPV Model
}

\author{
Qinghua Zhang, Lennart Ljung
}

\section{To cite this version:}

Qinghua Zhang, Lennart Ljung. From Structurally Independent Local LTI Models to LPV Model. Automatica, 2017, 84, pp.232-235. 10.1016/j.automatica.2017.06.006 . hal-01635096

\section{HAL Id: hal-01635096 https://inria.hal.science/hal-01635096}

Submitted on 14 Nov 2017

HAL is a multi-disciplinary open access archive for the deposit and dissemination of scientific research documents, whether they are published or not. The documents may come from teaching and research institutions in France or abroad, or from public or private research centers.
L'archive ouverte pluridisciplinaire HAL, est destinée au dépôt et à la diffusion de documents scientifiques de niveau recherche, publiés ou non, émanant des établissements d'enseignement et de recherche français ou étrangers, des laboratoires publics ou privés. 


\title{
From Structurally Independent Local LTI Models to LPV Model
}

\author{
Qinghua Zhang ${ }^{\mathrm{a}}$ Lennart Ljung ${ }^{\mathrm{b}}$ \\ ${ }^{\mathrm{a}}$ Inria-IFSTTAR, Campus de Beaulieu, 35042 Rennes Cedex, France qinghua.zhang@inria.fr \\ b Div. of Automatic Control, Linköping University, Sweden,ljung@isy.liu.se
}

\begin{abstract}
The local approach to linear parameter varying (LPV) system identification consists in interpolating individually estimated local linear time invariant (LTI) models corresponding to fixed values of the scheduling variable. It is shown in this paper that, without any global structural assumption of the considered LPV system, individually estimated local state-space LTI models do not contain sufficient information for determining similarity transformations making them coherent. It is possible to estimate these similarity transformations from input-output data under appropriate excitation conditions.
\end{abstract}

Key words: System identification, LPV model, coherent local linear models.

\section{Introduction}

Linear parameter varying (LPV) models provide an effective approach to handling nonlinear control systems $[14,9,6,11]$. Some successful methods for LPV system identification have been reported recently $[17,8,5,13$, $21,10]$. In the local approach to LPV system identification, interpolation is essential to establishing global models from a collection of locally estimated linear time invariant (LTI) models [14, 2, 1]. As LTI state-space models can be estimated in an arbitrary state basis, it is necessary to use a coherent collection of local models for the purpose of interpolation.

This paper is focused on the problem of making local state-space models coherent, without treating the interpolation step. Only state-space models are considered in this paper, as local model coherence is not relevant for other models. For shorter expressions, the words "statespace" will be omitted from terms like "local state-space model" and "LTI state-space model". In practice, interpolation is based on a finite set of local LTI models, each corresponding to a specific value of the scheduling variable $p(t)$, the main discussion of this paper is thus about the case where $p(t)$ evolves within a finite set, but its motivation is indeed with the perspective of interpolation for continuous values of $p(t)$.

It seems natural to transform all the local LTI models to some canonical form in order to make them coherent. The main purpose of this paper is to point out the fact that, in the local approach to LPV system identification, structurally independent local LTI models themselves do not contain sufficient information to determine similarity transformations making them coherent. However, locally estimated LTI models can be made coherent by making use of the information contained in some inputoutput data sequences across all the working points, notably with an algorithm initially introduced in the framework of piecewise linear hybrid systems [18, 20].

Preliminary results of this work have been presented in [20], which are completed in the present paper with a rigorous proof of the main result.

\section{Problem statement}

Let $u(t) \in \mathbb{R}^{q}$ and $y(t) \in \mathbb{R}^{s}$ be respectively the input and the output at discrete time instant $t=0,1,2, \ldots, p(t)$ be the scheduling variable evolving within a compact set $\square$. An LPV system is described by the state-space model

$$
\begin{aligned}
x(t+1) & =A(p(t)) x(t)+B(p(t)) u(t)+w(t) \\
y(t) & =C(p(t)) x(t)+D(p(t)) u(t)+v(t)
\end{aligned}
$$

where $x(t) \in \mathbb{R}^{n}$ is the state vector, $A(p(t)), B(p(t))$, $C(p(t)), D(p(t))$ are matrices of appropriate sizes depending on $p(t) \in \mathbb{\pi}$, and $w(t) \in \mathbb{R}^{n}, v(t) \in \mathbb{R}^{s}$ are state and output noises with covariance matrices $Q(p(t)), R(p(t))$.

Based on the fact that the LPV system (1) becomes an LTI system when the scheduling variable $p(t)$ is maintained at a fixed value, the following definitions aim at establishing a link between LPV and LTI models. 
Consider a set of $m$ LTI models indexed by the integer $i$ :

$$
\begin{aligned}
x(t+1) & =A_{i} x(t)+B_{i} u(t)+w(t) \\
y(t) & =C_{i} x(t)+D_{i} u(t)+v(t)
\end{aligned}
$$

characterized by matrices $A_{i}, B_{i}, C_{i}, D_{i}$ of appropriate sizes, and noise covariance matrices $Q_{i}$ and $R_{i}$.

The notation

$$
\sigma_{i} \triangleq\left(A_{i}, B_{i}, C_{i}, D_{i}, Q_{i}, R_{i}\right)
$$

will be used to denote the matrices characterizing the $i$ th local LTI model (2), or the LTI model itself by abuse of notation. The set of LTI models will be denoted by

$$
\Sigma=\left\{\sigma_{i}: i=1,2, \ldots, m\right\}
$$

Definition 1 A set of local LTI models

$$
\Sigma^{*}=\left\{\left(A_{i}^{*}, B_{i}^{*}, C_{i}^{*}, D_{i}^{*}, Q_{i}^{*}, R_{i}^{*}\right): i=1,2, \ldots, m\right\}
$$

is called a multi-snapshot of the LPV system (1) for

$$
p(t) \in \mathbb{P}=\left\{p_{1}, \ldots, p_{m}\right\} \subset \mathbb{\square},
$$

if

$$
\begin{aligned}
& A_{i}^{*}=A\left(p_{i}\right), B_{i}^{*}=B\left(p_{i}\right), C_{i}^{*}=C\left(p_{i}\right), \\
& D_{i}^{*}=D\left(p_{i}\right), Q_{i}^{*}=Q\left(p_{i}\right), R_{i}^{*}=R\left(p_{i}\right) .
\end{aligned}
$$

In the local approach to LPV system identification [14, $2,1]$, a set of locally estimated LTI models are interpolated to obtain a global model. As such local LTI models are typically estimated up to different and arbitrary similarity transformations, they do not constitute a multisnapshot of the underlying LPV system in the sense of Definition 1. It is thus important to make the local models "coherent" before the interpolation step.

What does mean a "coherent" set of local LTI models with the perspective of their interpolation? The following auxiliary definition will be helpful.

Definition 2 The input-output behavior of a set of local LTI models $\Sigma=\left\{\sigma_{1}, \ldots, \sigma_{m}\right\}$ is the input-output behavior of the multi-model switching system consisting of the same set of LTI models, such that the $i$-th LTI model $\sigma_{i}$ is active when $p(t)=p_{i} \in \mathbb{P}=\left\{p_{1}, \ldots, p_{m}\right\}$, and at every transition between two LTI models, the initial state of the new active model is equal to the final state of the previous active LTI model.

For example, following this definition, the input-output behavior of a multi-snapshot $\Sigma^{*}$ (see Definition 1) of an LPV system (1) is identical to input-output behavior the LPV system when $p(t)$ evolves within the restricted set P.
If the interpolation of a set of local LTI models is expected to describe correctly an LPV system for all sequences of $p(t)$ within $\square$, it should also be true in the particular case where $p(t)$ evolves within the restricted set $\mathbb{P}=\left\{p_{1}, \ldots, p_{m}\right\} \subset \mathbb{}$, including when $p(t)$ switches between different values within $\mathbb{P}$. It means that a "coherent" set of local LTI models should have the same inputoutput behavior as the underlying LPV system when $p(t) \in \mathbb{P}$, in the sense of Definition 2. This requirement will be satisfied by Definition 4 through Property 1 .

Definition 3 Two LTI models $\sigma \triangleq(A, B, C, D, Q, R)$ and $\tilde{\sigma} \triangleq(\tilde{A}, \tilde{B}, \tilde{C}, \tilde{D}, \tilde{Q}, \tilde{R})$ are related by a similarity transformation characterized by an invertible matrix $T \in$ $\mathbb{R}^{n \times n}$ and denoted as

$$
\sigma \stackrel{T}{\longrightarrow} \tilde{\sigma}
$$

if the matrices characterizing the two LTI models satisfy

$$
\begin{aligned}
& \tilde{A}=T A T^{-1}, \quad \tilde{B}=T B, \quad \tilde{C}=C T^{-1}, \\
& \tilde{D}=D, \quad \tilde{Q}=T Q T^{T}, \quad \tilde{R}=R .
\end{aligned}
$$

Definition 4 A set of LTI models

$$
\tilde{\Sigma}=\left\{\tilde{\sigma}_{i}: i=1,2, \ldots, m\right\}
$$

is said coherent with another set

$$
\Sigma=\left\{\sigma_{i}: i=1,2, \ldots, m\right\}
$$

if there exits an invertible transformation matrix $T \in$ $\mathbb{R}^{n \times n}$, common to the local models, such that

$$
\sigma_{i} \stackrel{T}{\longrightarrow} \tilde{\sigma}_{i} \text { for all } i=1, \ldots, m \text {. }
$$

This relationship between $\Sigma$ and $\tilde{\Sigma}$ is then denoted by

$$
\Sigma \stackrel{T}{\Longrightarrow} \tilde{\Sigma}
$$

The local LTI models $\tilde{\sigma}_{i}$ are simply said coherent when the reference model set $\Sigma$ is obvious.

The relevance of this definition is justified by the following property, as discussed before Definition 3.

Property 1 If a set of local models $\tilde{\Sigma}$ is coherent with the multi-snapshot $\Sigma^{*}$ of the LPV system (1), then $\tilde{\Sigma}$ has the same input-output behavior (in the sense of Definition 2) as that of the LPV system (1) with the scheduling variable $p(t)$ restricted to the finite set $\left\{p_{1}, \ldots, p_{m}\right\}$ and with appropriate initial states.

The proof of this property is trivial: under the assumed conditions, the LPV system (1) and its multi-snapshot $\Sigma^{*}$ have the same input-output behavior, and the states of $\Sigma^{*}$ and $\tilde{\Sigma}$ are related by the transformation matrix $T$. Property 1 is a necessary condition that a relevant definition of local model coherence should satisfy. It does 
not exclude other possible definitions, notably those based on $p$-dependent state transformation matrices $T(p)$. Such considerations, related to LPV system equivalent state-space representations as investigated in [3], would be out of the scope of this technical communiqué. In practice, local LTI models are estimated from a finite data sample subject to random uncertainties, thus the definition of coherent local models is understood in an approximative sense. If the estimation of each local model is consistent, then Definition 4 can also be understood for the limiting models when the data size for each local model estimation tends to infinity.

If some global structural assumptions of the matrix functions $A(p), B(p)$, etc. were assumed, then they could be used to make estimated local LTI models coherent. This paper is focused on structurally independent local LTI models, as defined below.

Definition 5 A set of local LTI models $\sigma_{i}$ are structurally dependent if their parametrizations are such that fixing the matrices $A_{i}, B_{i}, C_{i}, D_{i}, Q_{i}, R_{i}$ reduces the degrees of freedom of the matrices $A_{j}, B_{j}, C_{j}, D_{j}, Q_{j}, R_{j}$, for $j \neq i$; otherwise they are structurally independent.

For instance, if a set of local LTI models is parametrized such that all the matrices $A_{i}$ share an equal entry at the same position, say $A_{i}(1,1)=A_{j}(1,1)$ for all $i, j=$ $1,2, \ldots, m$, then the local models are structurally dependent. A less trivial counterexample will be given in Section 3.2 with equation (27).

\section{Making local LTI models coherent}

Apply different similarity transformations $\hat{T}_{i}$ to a set of local LTI models $\Sigma^{*}$, resulting in a transformed set of models $\hat{\Sigma}$, which is not coherent with $\Sigma^{*}$. Assume that neither $\hat{T}_{i}$ nor $\Sigma^{*}$ is known, is it possible to derive, solely from $\hat{\Sigma}$, a model set $\tilde{\Sigma}$ coherent with $\Sigma^{*}$ ?

\subsection{Structurally independent local LTI models}

Proposition 1 Given a set of structurally independent LTI models

$$
\Sigma^{*}=\left\{\sigma_{i}^{*}: i=1,2, \ldots, m\right\},
$$

assume that a set of arbitrary unknown invertible matrices

$$
\left\{\hat{T}_{i} \in \mathbb{R}^{n \times n}: i=1,2, \ldots, m\right\}
$$

transform the LTI models

$$
\sigma_{i}^{*} \hat{T}_{i} \hat{\sigma}_{i}
$$

resulting in a set of transformed models

$$
\hat{\Sigma}=\left\{\hat{\sigma}_{i}: i=1,2, \ldots, m\right\},
$$

then it is impossible to determine invertible matrices $\tilde{T}_{i} \in \mathbb{R}^{n \times n}$ (not necessarily equal to $\hat{T}_{i}$ ) for $i=1, \ldots, m$, solely based on the set of transformed models $\hat{\Sigma}$, such that the further transformed set of models

$$
\tilde{\Sigma}=\left\{\tilde{\sigma}_{i}: i=1,2, \ldots, m\right\}
$$

through

$$
\hat{\sigma}_{i \stackrel{T_{i}}{\longrightarrow}} \tilde{\sigma}_{i}
$$

is coherent with the original set $\Sigma^{*}$, or more precisely,

$$
\Sigma^{*} \stackrel{T}{\Longrightarrow} \tilde{\Sigma}
$$

for some unknown $T \in \mathbb{R}^{n \times n}$.

Proof. The proof proceeds by contradiction, starting by assuming the contrary of Proposition 1, in which the word "impossible" is replaced by "possible", or more compactly, "solely based on $\hat{\Sigma}$, the original model set $\Sigma^{*}$ can be determined up to an unknown transformation matrix $T$ common to all local LTI models". As the matrix $T \in \mathbb{R}^{n \times n}$ can be any invertible matrix, what is determined from $\hat{\Sigma}$, is a set of (infinitely many) sets of LTI models, which will be denoted by $\mathbb{S}(\hat{\Sigma})$ in what follows. The contrary of Proposition 1 then means

$$
\mathbb{S}(\hat{\Sigma})=\left\{\Sigma: \Sigma^{*} \stackrel{T}{\Longrightarrow} \Sigma, T \in \mathbb{R}^{n \times n}, \operatorname{det}(T) \neq 0\right\}
$$

Within this set, the particular element $\Sigma^{*}$ corresponds to the identity transformation matrix $T$, hence

$$
\Sigma^{*} \in \mathbb{S}(\hat{\Sigma})
$$

Consider a set of $m$ invertible and pairwise distinct matrices $S_{i} \in \mathbb{R}^{n \times n}, i=1,2, \ldots, m$ and a model set

$$
\Sigma^{\prime}=\left\{\sigma_{i}^{\prime}: i=1,2, \ldots, m\right\}
$$

such that

$$
\sigma_{i}^{\prime} \stackrel{S_{i}}{\longrightarrow} \sigma_{i}^{*}
$$

for $i=1,2, \ldots, m$. The two transformations (22) and (14) then imply

$$
\sigma_{i \stackrel{T_{i}}{\prime}}^{\prime} \hat{\sigma}_{i}
$$

with $\hat{T}_{i}^{\prime}=\hat{T}_{i} S_{i}$, for $i=1,2, \ldots, m$. This result means that the model set $\hat{\Sigma}$ originating from $\Sigma^{*}$ through transformations (14) could also have originated from $\Sigma^{\prime}$ through transformations (23). Therefore, following the same reasoning as at the beginning of this proof, that has led to $\Sigma^{*} \in \mathbb{S}(\hat{\Sigma})$, it yields $\Sigma^{\prime} \in \mathbb{S}(\hat{\Sigma})$. This result 
and (19) imply that $\Sigma^{*} \stackrel{T}{\Longrightarrow} \Sigma^{\prime}$ for some invertible $T$. In terms of the LTI models composing $\Sigma^{*}$ and $\Sigma^{\prime}$, it means

$$
\sigma_{i}^{*} \stackrel{T}{\longrightarrow} \sigma_{i}^{\prime}
$$

with a common $T$ for all $i=1,2, \ldots, m$. This result is clearly in contradiction with $(22)$, where $S_{i}$ are pairwise distinct matrices. This contradiction invalidates the assumption made at the beginning of this proof, hence proving Proposition 1.

\subsection{Examples based on global structural assumptions}

The result of Proposition 1 may seem in contradiction with some known methods for making local LTI models coherent. In fact, these methods assume (implicitly) some particular structure of the matrix-valued functions $A(p), B(p)$ etc., therefore, the local LTI models are not structurally independent in the sense of Definition 5. To better clarify the situations, some examples are recalled below, by pointing out their particular global structural assumptions.

\subsubsection{Coherent LTI models based on canonical forms}

In order to make local LTI models coherent, a natural $i d e a$ is to find similarity transformations of the local LTI models leading to some LTI canonical state-space form, or to estimate local LTI models directly in such a form $[19,12,7,2]$. This practice assumes that the local LTI models are coherent when they are all transformed into the same LTI canonical form ${ }^{1}$.

For the sake of presentation simplicity, consider the case of single-input-single-output (SISO) models. In the controllable form, the $m$ local models involve, for $i=1,2, \ldots, m$,

$$
\tilde{A}_{i}=\left[\begin{array}{ccccc}
0 & 1 & 0 & \cdots & 0 \\
0 & 0 & 1 & \cdots & 0 \\
\vdots & \vdots & \vdots & \cdots & \vdots \\
a_{i}^{(1)} & a_{i}^{(2)} & a_{i}^{(3)} & \cdots & a_{i}^{(n)}
\end{array}\right], \quad \tilde{B}_{i}=\left[\begin{array}{c}
0 \\
0 \\
0 \\
\vdots \\
1
\end{array}\right]
$$

The assumption that the local LTI models in this canonical form are coherent implies that there exists a common invertible matrix $T \in \mathbb{R}^{n \times n}$ such that, for all $i=$ $1,2, \ldots, m, \tilde{A}_{i}=T A_{i}^{*} T^{-1}$. Let $\sqcap(M)$ denote the sub

\footnotetext{
$\overline{1}$ Transforming the local LTI models to the same LTI canonical form does not lead to a canonical form of the underlying LPV system, as pointed out in [15].
}

matrix of $M$ excluding its last row, then

$$
\sqcap\left(T A_{i}^{*} T^{-1}\right)=\left[\begin{array}{ccccc}
0 & 1 & 0 & \cdots & 0 \\
\vdots & \vdots & \vdots & \cdots & \vdots \\
0 & 0 & 0 & \cdots & 1
\end{array}\right], \quad \forall i=1,2, \ldots, m .
$$

By assuming that $\sqcap\left(T A_{i}^{*} T^{-1}\right)$ are equal to the same matrix for all $i=1,2, \ldots, m$, the local LTI models are not structurally independent in the sense of Definition 5.

\subsubsection{Coherent LTI models based on the observability matrix}

In [1] another method is proposed to make local LTI models coherent, in a framework more general than the one of the present paper. In the particular case of SISO observable systems, this method consists in finding different similarity transformations so that the $m$ transformed local LTI models all have the same observability matrix. It implies the assumption that the local LTI systems composing a multi-snapshot of the underlying LPV system all have the same observability matrix, or more explicitly,

$$
C_{i} A_{i}^{s}=C_{j} A_{j}^{s}
$$

for all $i, j \in\{1,2, \ldots, m\}$ and $s \in\{0,1, \ldots, n-1\}$. Again the local LTI models are not structurally independent in the sense of Definition 5.

Remark. This observability matrix-based method is incompatible with the previously presented example of canonical form-based method, in the sense that in general the two resulting model sets are not coherent with each other. Moreover, the canonical observable formbased method is incompatible with the canonical controllable form-based method, and similarly the observability matrix-based method is incompatible with the controllability matrix-based method. These incompatibilities between "natural" methods illustrate the fact that there is no generally natural global structural assumption for making estimated local LTI models coherent.

\section{Data-based transformations for coherent LTI models}

In order to make local LTI models coherent, as an alternative to global structural assumptions, the information contained in some data sequences can be used.

The idea is based on Property 1 that is satisfied by coherent local LTI models. In particular, the state trajectory "continuity" in Definition 2 is essential: when the scheduling variable $p(t)$ switches from one value to another within $\mathbb{P}$, the local LTI model switches accordingly, but the initial state of the new active LTI model is equal to the final state of the previous active LTI model. 
Given a data set $\mathbb{D}=\{(p(t), u(t), y(t)): t=1,2, \ldots, N\}$ with $p(t) \in \mathbb{P}$, start by segmenting the data sequence into pieces of constant $p(t)$ value. If the local LTI models are estimated from pieces of this data set, the whole state trajectory on each data segment can be obtained as a by-product of the identification procedure [16]. If the local LTI models have been estimated from another data set, then the initial state and the whole state trajectory on each data segment of $\mathbb{D}$ can be estimated with the already estimated local LTI models [4]. Similarity transformations of the local LTI models are then determined so that, when the local LTI model switches, the initial state of the new active LTI model is equal to the final state of the previous active LTI model.

A complete data-based algorithm for making local LTI models coherent has been introduced in [18] in the framework of piecewise linear hybrid systems. A variant algorithm has been presented in [20].

\section{Conclusion}

It has been shown in this paper that structurally independent local LTI models estimated from data collected with fixed scheduling variable of an LPV system do not contain sufficient information to make themselves coherent with the perspective of LPV model interpolation. Nevertheless, well designed data sequences can be used to address this problem.

\section{References}

[1] J. De Caigny, R. Pintelon, J.F. Camino, and J. Swevers. Interpolated modeling of LPV systems. IEEE Trans. on Control System Technology, 22(6):2232 2246, 2014.

[2] Jan De Caigny, Juan F. Camino, and Jan Swevers. Interpolation-based modeling of MIMO LPV systems. IEEE Trans. on Control System Technology, 19(1):46-63, 2011.

[3] B Kulcsar and Roland Tóth. On the similarity state transformation for linear parameter-varying systems. In 18th IFAC World Congress, Milano, 2011.

[4] Lennart Ljung. System Identification - Theory for the User. Prentice-Hall, 2nd edition edition, 1999.

[5] P. Lopes dos Santos, T.-P. Azevedo-Perdicoúlis, J.A. Ramos, J.L. Martins de Carvalho, G. Jank, and J. Milhinhos. An LPV modeling and identification approach to leakage detection in high pressure natural gas transportation networks. IEEE Trans. on Control System Technology, 19(1):77-92, January 2011.

[6] Paulo Lopes dos Santos, Teresa Paula Azevedo Perdicoulis, Carlo Novara, Jose A Ramos, and Daniel E Rivera, editors. Linear ParameterVarying System Identification. World Scientific Publishing Company, New Jersey, 2012.

[7] Marco Lovera and Guillaume Mercere. Identification for gain-scheduling: a balanced subspace approach. In American Control Conference, pages 858-863, New York City, 2007.
[8] Guillaume Mercere, Halldor Palsson, and Thierry Poinot. Continuous-time linear parameter-varying identification of a cross: A local approach. IEEE Trans. on Control System Technology, 19(1):64-76, 2011.

[9] Javad Mohammadpour and Carsten W. Scherer, editors. Control of Linear Parameter Varying Systems with Applications. Springer, New York, 2012.

[10] D. Piga, P. B. Cox, R. Toth, and V. Laurain. Identification of LPV models from noise-corrupted scheduling parameter and output observations. Automatica, To appear, 2015.

[11] Olivier Sename, Peter Gaspar, and Jozsef Bokor, editors. Robust Control and Linear Parameter Varying Approaches. Springer, Berlin, 2013.

[12] M. Steinbuch, R. van de Molengraft, and A. van der Voort. Experimental modelling and LPV control of a motion system. In American Control Conference, volume 2, pages 1374-1379, Denver, 2003.

[13] R. Tóth, V. Laurain, M. Gilson, and H. Garnier. Instrumental variable scheme for closed-loop LPV model identification. Automatica, 48(9):2314-2320, 2012.

[14] Roland Tóth. Modeling and Identification of Linear Parameter-Varying Systems. Springer, 2010.

[15] Roland Tóth, Federico Felici, Peter S. C. Heuberger, and Paul M. J. Van den Hof. Discrete time LPV I/O and state space representations, differences of behavior and pitfalls of interpolation. In European Control Conference, pages 5418-5425, Kos, 2007.

[16] P. Van Overschee and B. De Moor. Subspace Identification for Linear Systems: Theory, Implementation Applications. Springer-Verlag, 1996.

[17] Jan-Willem Van Wingerden and Michel Verhaegen. Subspace identification of bilinear and LPV systems for open- and closed-loop data. Automatica, 45(2):372-381, 2009.

[18] Vincent Verdult and Michel Verhaegen. Subspace identification of piecewise linear systems. In 43rd IEEE Conference on Decision and Control, Atlantis, Paradise Island, Bahamas, 2004.

[19] Jeremy Hoyt Yung. Gain scheduling for geometrically nonlinear flexible space structures. $\mathrm{PhD}$ thesis, MIT, Cambridge, 2002.

[20] Qinghua Zhang and Lennart Ljung. LPV System Common State Basis Estimation from Independent Local LTI Models. In 17th IFAC Symposium on System Identification (SYSID), Beijing, France, October 2015.

[21] Yu Zhao, Biao Huang, Hongye Su, and Jian Chu. Prediction error method for identification of LPV models. Journal of Process Control, 22(1):180-193, 2012. 